\title{
Lumen
}

Selected Proceedings from the Canadian Society for Eighteenth-Century Studies

\section{Machines à rêves? L’imaginaire du théâtre classique d'après les planches de l'Encyclopédie}

\section{Guy Spielmann}

Volume 25, 2006

URI : https://id.erudit.org/iderudit/1012080ar

DOI : https://doi.org/10.7202/1012080ar

Aller au sommaire du numéro

Éditeur(s)

Canadian Society for Eighteenth-Century Studies / Société canadienne d'étude du dix-huitième siècle

ISSN

1209-3696 (imprimé)

1927-8284 (numérique)

Découvrir la revue

Citer cet article

Spielmann, G. (2006). Machines à rêves? L'imaginaire du théâtre classique

d'après les planches de l'Encyclopédie . Lumen, 25, 103-120.

https://doi.org/10.7202/1012080ar

Copyright (C Canadian Society for Eighteenth-Century Studies / Sociéte canadienne d'étude du dix-huitième siècle, 2006
Ce document est protégé par la loi sur le droit d'auteur. L'utilisation des services d'Érudit (y compris la reproduction) est assujettie à sa politique d'utilisation que vous pouvez consulter en ligne.

https://apropos.erudit.org/fr/usagers/politique-dutilisation/ 


\section{Machines à rêves ? L'imaginaire du théâtre classique d'après les planches de l'Encyclopédie}

En consacrant un volume de planches au théâtre et à ses machines, les éditeurs de l'Encyclopédie se sont-ils interrogés sur la forme que ces illustrations devaient prendre, sur la manière dont on peut représenter «le théâtre», non pas en tant que genre littéraire, mais en tant que pratique technique ? Que fallait-il en montrer, et dans quel but? Même si l'on ne trouve nulle part de réponse explicite à de telles questions, un examen attentif et critique de la série de 89 gravures que contient la «Neuvième livraison» des planches de l'Encyclopédie $(1772)^{1}$ nous permet de cerner le sens des choix qui ont été opérés, et par là de mieux comprendre les enjeux de cette entreprise, sutout si on la compare à l'iconographie antérieure. Bien que le parti-pris de fournir principalement des illustrations schématiques de salles de spectacle et de machines utilisées à l'opéra suggère une vision positiviste, voire mécaniste de l'art dramatique, l'ensemble des planches constitue aussi un document précieux sur l'imaginaire du théâtre classique, bien que, comme nous le constaterons, la valeur exacte de ces images se révèle différente de ce qu'on aurait pu croire à première vue.

La prédominance historique d'une approche littéraire du théâtre a pu laisser penser que cet imaginaire se construisait et s'exprimait principalement par le truchement du langage - du logos, de l'histoire, du discours - , tandis que dans le cas de l'opéra, où c'est le point de vue musicologique qui a traditionnellement prévalu, cet imaginaire a été le

1 «THÉATRES» et «MACHINES DE THÉATRE, Contenant quarante-neuf Planches à cause de quatorze doubles \& de quatre triples. Dessinées \& expliquées par M. Radel, Pensionnaire du Roi, \& Architecte - Expert, sous la direction de M. Giraud, Architecte des Menus Plaisirs, \& Machiniste de l'Opéra de Paris ", dans Denis Diderot et Jean le Rond D'Alembert (éditeurs), Encyclopédie, ou Dictionnaire raisonné des sciences, des arts et des métiers, Paris, Briasson, David l'aîné, Le Breton et Durand, «Neuvième livraison ou dixième volume» (t. 27), 1772. 
plus souvent défini en termes d'harmonie, de tonalité, de couleur, de mélodie et de rythme. Mais pour le spectateur du XVIII ${ }^{\mathrm{e}}$ siècle, une troisième dimension s'ajoutait à ces deux premières, sans qu'on puisse formellement déterminer aujourd'hui si l'une d'entre elles primait sur les autres : celle de l'imaginaire visuel, qui se nourrissait d'abord de l'expérience de représentations vues et revues, et comparées entre elles².

La plus évidente constatation qui s'impose à nous, c'est que la mise en scène et la scénographie du théâtre à l'Italienne de l'âge baroque obéissaient à des règles d'esthétique et de mécanique qui, pour n'être pas écrites, s'appliquaient de manière bien plus contraignante que celles de la poétique. En effet, tandis que les principes fondamentaux de la composition dramatique (les trois unités, la vraisemblance, les bienséances, etc.) restaient sujets à de nombreuses entorses, y compris de la part des auteurs les plus confirmés, l'usage des décors, des machines et des costumes était régi par des lois qui s'appliquaient de façon extrêmement stricte, quasiment sans exception - tout en demeurant largement implicites. Alors que de nos jours de telles lois sont formulées dans des manifestes, analysées par les chercheurs et enseignées dans les écoles, elles relevaient alors pour l'essentiel d'une tradition orale, transmise par l'apprentissage plutôt que par l'enseignement.

Rappelons que ce que nous nommons actuellement les arts de la scène n'étaient pas réputés appartenir au domaine des beaux-arts, qui faisaient l'objet d'un discours savant et d'une supervision officielle confiée à une académie ; mais justement, «l'une des intentions majeures» de L'Encyclopédie était $\mathrm{d}^{\prime}$ ' offrir une 'decriptions des....arts mécaniques' par opposition aux 'arts libéraux' dont la tradition humaniste avait exclusivement célébré la gloire ${ }^{3}$ ». Comme l'a souligné Jacques Proust, Diderot, dans

2 On trouve un excellent document sur ce phénomène dans le Journal de Charles Collé, à peu près contemporain de l'Encyclopédie, où l'auteur tient la chronique non seulement des pièces nouvellement créées, mais aussi des reprises - avec les divers changements qui les accompagnent, et les réactions non moins diverses du public relatant ainsi l'expérience d'une fréquentation continue des spectacles de Paris.Voir Journal et mémoires de Charles Collé, sur les hommes de lettres, les ouvrages dramatiques et les événements les plus mémorables du règne de Louis XV (1748-1772), Nouvelle édition augmentée de fragments inédits, éd. Honoré Bonhomme, Paris, Firmin Didot frères, fils et Cie., 3 vols., 1868.

3 Robert Mauzi, «Une souveraineté éphémère», dans L'Univers de l'Encyclopédie, Paris, Les Libraires Associés, coll. «Images d'une civilisation»,1964, p. 19. Diderot expliquait dans le Prospectus définitif de l'Encyclopédie (1750) que «Toute la matière de l'Encyclopédie peut se réduire à trois chefs : les sciences, les arts libéraux et les arts mécaniques». 
l'article "Art», traduit cette volonté par une «insistance [...] sur l'égale dignité des arts libéraux et des arts manuels ${ }^{4} »$.

De fait, "feinteurs» et machinistes, soit avaient débuté comme ingénieurs, soit venaient des arts appliqués ; ils pouvaient être "peintreartificier» (décorateur), comme Buffequin ${ }^{5}$; le plus influent d'entre eux, celui qui définit presque à lui seul le style classique, Jean Berain, était à l'origine maître arquebusier : outre des décors et des costumes de scène, on lui doit une œuvre d'une immense variété où le mobilier côtoie les navires ${ }^{6}$. Mais les véritables artistes-peintres, à de rarissimes exceptions près (Boucher en particulier ${ }^{7}$ ) ne prêtèrent jamais leur talent aux productions dramatiques, même lorsque par ailleurs ils traitaient fréquemment, comme Gillot et Watteau, des sujets tirés du théâtre.

Si donc nul ne contestait le fait qu'une représentation théâtrale constitue, dans son ensemble, une manifestation artistique, ses composantes matérielles restaient considérées comme purement techniques. C'est sans doute pourquoi les savoirs qu'elles impliquent ont fait l'objet d'écrits relativement peu nombreux, compte tenu de l'importance de l'activité dramatique en Europe de la fin du XVI ${ }^{e}$ au milieu du XVIII siècle ; les traités sur le sujet ont en commun de dresser l'état des pratiques sans proposer de visée théorique novatrice, ni formuler explicitement de programme esthétique. Les plus connus, ceux de Serlio (1545), de Vignola (1583), de Troili (1672) et de Pozzo (1693) ${ }^{8}$, restent

4 Jacques Proust, "Présentation " de L'Encyclopédie Diderot et d'Alembert : planches et commentaires, Paris, Hachette, 1985, p. 13 : «Rendons enfin aux Artistes la justice qui leur est dûe. Les Arts libéraux se sont assez chantés eux-mêmes; ils pourroient employer maintenant ce qu'ils ont de voix à célébrer les Arts méchaniques. C'est aux Arts libéraux à tirer les Arts méchaniques de l'avilissement où le préjugé les a tenus si longtems; c'est à la protection des rois à les garantir d'une indigence où ils languissent encore. Les Artisans se sont crus méprisables, parce qu'on les a méprisés ; apprenons-leur à mieux penser d'eux-mêmes : c'est le seul moyen d'en obtenir des productions plus parfaites».

5 Voir Hélène Visentin, «Décorateur à la Cour et à la Ville : un artisan de la scène nommé Georges Buffequin (1585 ?-1641)", XVII' siècle, n. 195, 1997, 325-339.

6 Voir Jérôme de la Gorce, Berain, dessinateur du Roi Soleil, Paris, Herscher, 1986. On sait aussi que la configuration du dispositif machinique propre au théâtre à l'Italienne doit beaucoup à l'architecture de marine, d'où les premiers scénographes modernes étaient souvent issus.

7 Boucher exécuta des dessins de décors et de costumes pour l'Opéra à partir de 1741, ainsi que pour l'Opéra-Comique à partir de 1743. Voir François Boucher, 1703-1770, Paris, Éditions de la Réunion des musées nationaux, 1986, p. 216-217.

8 Sebastiano Serlio, «Trattato sopra le Scene», dans Il secondo libro di prospettiva, publié 
principalement attachés à la tradition vitruvienne, c'est-à-dire à des conceptions remontant à l'antiquité et agrémentées des effets de perspective inventés à la Renaissance ${ }^{9}$. Celui de Sabbattini $(1638)^{10}$ ne représentait une innovation que dans la mesure où, pour la première fois, un homme de l'art (et non plus un architecte ou un peintre) livrait dans le détail des indications précises pour obtenir des effets scéniques donnés ; Duvignaud voyait à tort dans ce catalogue le manifeste d'une "réforme» promulguée par un maître dont Torelli et Vigarani auraient été les «élèves ${ }^{11}$ ».

Signalons encore l'œuvre de Furttenbach, qui donne des explications détaillées sur divers effets (vagues, nuages, monstres, éclairages) et expose clairement, mais très tardivement (1663) le dispositif de changement de décors par périactes, déjà utilisé dans la Grèce antique ${ }^{12}$, et enfin celle de Galli-Bibiena $(1711)^{13}$, où l'on trouve la seule véritable innovation marquante enregistrée depuis la «roue de Torelli» des années

avec Il primo libro d'architettura, di Sabastiano Serlio, bolognese, Paris, Jean Barbé, 1545 ; Jacomo Barozzi Vignola, Le due regole della prospetiva pratica..., Rome, Zanetti, 1583 ; Giulio Troili, Paradossi per pratticare la prospettiva senza saperla..., Bologne, Peri, 1672 ; Andrea Pozzo, Prospettiva de' pittori e architetti, Rome, Boëmo, 1693.

9 Le De architectura de Marcus Vitruvius Pollio, architecte romain de l'époque d'Auguste, avait été réédité en 1511, puis traduit par Daniele Barbaro avec des planches d'Andrea Palladio (Venise, 1556) ; ce traité eut un impact comparable à celui de la Poétique d'Aristote dans le domaine dramatique. A l'époque de l'Encyclopédie, le terme "scénographie» ne désignait encore que «la représentation d'un corps en perspective sur un plan ; c'est-à-dire la représentation de ce corps dans toutes ses dimensions, tel qu'il paroît à l'oeil» (t. 14, 1765).

10 Nicola Sabbattini, Pratica di fabricar scene e machine ne' teatri, Pesaro, 1638. Reproduit en facsimile dans Scene e macchine teatrali della commedia dell'arte e della scenotecnica barocca con i disegni originali, éd. Alberto Perrini, Rome, E \& A editori, 1989. Version française : Pratique pour fabriquer des scènes et machines de théâtre, trad. Maria et René Canavaggia, avec une préface de Louis Jouvet, Paris, Ides et Calendes, 1942.

11 Jean Duvignaud, Théâtre et société, Paris, Denoël-Gonthier, 1970, ch. 3, «Sabbattini et la boîte close», p. 67-82. Hypothèse d'autant moins convaincante que Sabbattini ne travaillait pas dans l'un des grands centres d'innovation technique du premier XVII siècle, dont au premier chef Venise.

12 Joseph Furttenbach, Architectura Recreationis, Augsbourg, Schultes, 1640, et Manhafter Kunstspiegel (Le Noble Miroir de l'art), Augsbourg, 1663. Voir The Renaissance Stage : Documents of Serlio, Sabbattini, and Furttenbach, éd. Barnard Hewitt, Coral Gables, University of Miami, 1958.

13 Ferdinando Galli-Bibiena, L'Architettura civile preparata sù la geometria, e ridotta alle prospettive. Considerazioni pratiche, Parme, Monti, 1711. 
$1640^{14}$, le plan du décor en perspective décalée, la scena veduta per angolo, dont on trouve un exemple sur la planche de l'Encyclopédie intitulée «Salle de Spectacle de la Comédie Françoise [...] coupe en travers ${ }^{15}$ ». Ces ouvrages offrent aux lecteurs des illustrations qui relèvent plus généralement du plan ou du schéma, sans recherche esthétique particulière, tandis que, par ailleurs, la riche tradition d'iconographie théâtrale qui s'était développée parallèlement (Callot, Spada, Rabel, Bonnart, Gillot, Silvestre, Le Pautre, Moreau, Watteau...) cherchait moins à représenter la réalité scénique qu'une vision idéalisée de l'action dramatique, en ne fournissant aucun indice quant aux techniques utilisées.

Où se situe l'Encyclopédie ? Elle se rattache à la première catégorie, apparemment, puisłúu'elle s'en tient à l'architecture des salles (les 38 planches de la section "Théâtre») et au détail des dispositifs mécaniques (les 49 planches de la section «Machines de théâtre»). Si de tels documents ne semblent pas d'abord s'adresser à notre sens du rêve ou de l'esthétique, c'est peut-être parce que nous les considérons dans une perspective post-romantique où la poésie s'oppose à la technique. Pourtant, ce volume offre une vision raisonnée des moyens dont diposait alors l'Opéra de Paris (c'est-à-dire le nec plus ultra en matière d'équipement mécanique) pour concrétiser sur la scène l'univers merveilleux du théâtre baroque, où l'invention - au sens rhétorique - reposait avant tout sur une savante combinatoire d'éléments prédéterminés, qu'il $\mathrm{s}^{\prime}$ agisse de l'écriture ou des effets scéniques ${ }^{16}$.

Même si en 1772 on n'a pas voulu se contenter de reprendre tels quels les dessins et schémas déjà publiés dans les traités antérieurs, les planches originales reflètent un état des techniques qui a fort peu évolué au cours des deux siècles précédents : les paramètres de la scène à

14 Dispositif permettant d'opérer simultanément plusieurs panneaux coulissants, que Torelli avait apporté de Venise à Paris, où Mazarin le fit venir en 1645 pour assurer la scénographie des divertissements de cour. Voir Per Bjustrom, Giacomo Torelli and Baroque Stage Design, Stockholm, Nationalmuseum, 1961.

15 «Théâtres», Planche IV.

16 Sur ces questions, voir notre article «Poétique(s) du merveilleux dans les arts du spectacle aux XVII ${ }^{\mathrm{e}}$ et XVIII ${ }^{\mathrm{e}}$ siècles», dans David Wetsel, Frédéric Canovas et al (éd.), Le Merveilleux au XVII siècle, Tübingen, Narr Verlag, 2003, vol. III, p. 227-240, dont la version illustrée peut être consultée sur le site www9.georgetown.edu/faculty/ spielmag/articles. Voir également les travaux d'Hélène Visentin, notamment «Le Théâtre à machines : succès majeur pour un genre mineur», Littératures Classiques, n. 51, 2004, 205-222, et l'ouvrage en préparation Le Théâtre à machines en France à l'âge classique. Introduction à l'histoire et à la poétique du théâtre à merveilles (1585-1680). 
l'Italienne avaient été fixés pour l'essentiel avant même le début du XVII ${ }^{\mathrm{e}}$ siècle, et matérialisés dans des édifices comme le Teatro olimpico de Vicenza (construit en 1585 par Vincento Scamozzi sur les plans d'Andrea Palladio) et le Teatro farnese de Parme (conçu par Gianbattista Aleotti en 1628). Les théâtres représentés dans l'Encyclopédie (Turin, Stuttgart, Comédie-Française, Montpellier, Tuileries...) n'offrent guère que des variations sur ce modèle, qui ne sera pas dépassé avant la toute fin du XIX ${ }^{e}$ siècle. Il fallut en effet attendre les alternatives radicales proposées par des architectes comme Appia et Gropius, par des décorateurs comme Gordon Craig, ou par les créateurs eux-mêmes (comme Wagner pour Bayreuth), conjuguées aux progrès techniques (l'électricité notamment), pour que l'agencement des lieux de spectacle pût commencer à évoluer.

Même le grand théâtre de Bordeaux, édifié par Victor Louis au moment même où paraissait le dixième volume de planches en 1772 , et célébré en son temps pour ses innovations ${ }^{17}$, se conformait encore à ce dispositif, dont la vue en coupe de la Comédie de Lyon (planches $\mathrm{M}$ et $\mathrm{N}$ ) donne un échantillon archétypique avec son cadre de scène et son décor en perspective centrale symétrique. Dans le contexte français, où, jusqu'à la fin du XVII ${ }^{e}$ siècle, les salles de théâtre avaient toujours été aménagées dans des jeux de paume ou dans des ailes de châteaux, la nouveauté résidait surtout à concevoir et construire un bâtiment dans le but d'y donner des représentations. Nul ne songeait alors à contester sérieusement ce que Diderot, quelque vingt ans plus tôt, n'hésitait pas à présenter dans les Entretiens sur le Fils Naturel comme la configuration idéalement propice à la stimulation de l'imaginaire dramatique :

Moi - Qu'appelez-vous changer la scène?

Dorval - En ôter tout ce qui resserre un lieu déjà trop étroit ; avoir des décorations ; pouvoir exécuter d'autres tableaux que ceux qu'on voit depuis cent ans ; en un mot, transporter au théâtre le salon de Clairville, comme il est.

Moi - Il est donc bien important d'avoir une scène ?

Dorval - Sans doute. Songez que le spectacle français comporte autant de décorations que le théâtre lyrique, et qu'il en offrirait de plus agréables, parce que le monde enchanté peut amuser des enfants, et qu'il n'y a que

17 Voir Paul Roudie et Philippe Rouyer (éd.), Victor Louis et le théâtre : Scénographie, mise en scène et architecture théâtrales aux XVIII ${ }^{\mathrm{e}}$ et XIX ${ }^{\mathrm{e}}$ siècles, Paris, CNRS, 1982. 
le monde réel qui plaise à la raison... Faute de scène, on n'imaginera rien. Les hommes qui auront du génie se dégoûteront ; les auteurs médiocres réussiront par une imitation servile; on s'attachera de plus en plus à de petites bienséances ; et le goût national s'appauvrira... Avez-vous vu la salle de Lyon? Je ne demanderais qu'un pareil monument dans la capitale, pour faire éclore une multitude de poèmes, et produire peut-être quelques genres nouveaux ${ }^{18}$.

La vocation des planches sur les théâtres dans l'Encyclopédie ne pouvait toutefois se limiter à présenter au lecteur un type de salle de spectacle qu'il connaissait probablement déjà ; il s'agissait de lui en faire voir les dessous - littéralement - et de l'aider à comprendre comment fonctionnait la boîte à illusions. Notons déjà le caractère paradoxal de cette démarche, puisque le principe de la scène à l'Italienne exige une recherche de vraisemblance dans la réalisation des effets qui implique l'effacement des techniques utilisées : impossible donc de livrer au lecteur des planches analogues à celles qui illustraient d'autres métiers comme la sellerie ou la ferronnerie, et ce d'autant plus que les secrets des ingénieurs et des décorateurs étaient jalousement conservés, et que les encyclopédistes ne poussèrent pas leur zèle jusqu'à vouloir les révéler, bien qu'on soit tenté d'accorder par principe à Diderot et ses confrères le crédit d'avoir dissipé l'obscurantisme des siècles précédents :

Autre illusion, autre noblesse, si l'on veut, de l'Encyclopédie, autre originalité en tout cas par rapport à ses précurseurs. Les traités de technologie du XVI et du XVII ${ }^{e}$ siècle avaient toujours un peu l'air de transmettre des secrets. Mais transmettre n'est pas les révéler ni les dissoudre. C'est seulement leur permettre de survivre en tant que secrets et de se propager obscurément dans cette pénombre où végètent toutes les magies ${ }^{19}$.

Certes, conformément à l'esprit encyclopédiste, l'approche parait de prime abord rationaliste et didactique, voire même démystifiante par sa volonté de dévoiler au spectateur devenu lecteur les rouages de machines destinées à provoquer l'émerveillement par la mise en scène de prodiges inexplicables : dans les années 1640, à l'arrivée en France de techniques venues d'Italie, d'Aubignac s'était indigné de la crédulité du

18 Denis Diderot, Entretiens sur le Fils Naturel (1757), dans CEuvres esthétiques, éd. Paul Vernière, Paris, Garnier, 1966, p. 112-113.

19 R. Mauzi, «Une souveraineté éphémère», loc. cit., p. 20. 
public, abusé par les effets spéciaux au point de croire qu'on y employait la magie plutôt que la mécanique ${ }^{20}$; un demi-siècle plus tard, Fontenelle prenait pour exemple l'un des plus célèbres effets spéciaux de l'opéra pour illustrer les progrès de la philosophie mécaniste, en des termes singulièrement prémonitoires qui semblent annoncer précisément la démarche qui préside à l'élaboration des planches de l'Encyclopédie :

Sur cela je me figure toûjours que la nature est un grand spectacle qui ressemble à celuy de l'opera. Du lieu où vous estes à l'opera, vous ne voyez pas le theatre tout-à-fait comme il est ; on a disposé les décorations et les machines pour faire de loin un effet agreable, et on cache à vostre veuë ces rouës et ces contrepoids qui font tous les mouvemens. Aussi ne vous embarassez-vous guere de deviner comment tout cela jouë. Il n'y a peut-estre que quelque machiniste caché dans le parterre, qui s'inquiete d'un vol qui luy aura paru extraordinaire, et qui veut absolument démesler comment ce vol a esté executé. Vous voyez bien que ce machiniste-là est assez fait comme les philosophes. Mais ce qui à l'égard des philosophes augmente la difficulté, c'est que dans les machines que la nature presente à nos yeux, les cordes sont parfaitement bien cachées, et elles le sont si bien, qu'on a esté long-temps à deviner ce qui causoit les mouvemens de l'univers ; car representez-vous tous les sages à l'opera, ces Pithagores, ces Platons, ces Aristotes, et tous ces gens dont le nom fait aujourd'huy tant de bruit à nos oreilles. Supposons qu'ils voyoient le vol de Phaëton que les vents enlevent, qu'ils ne pouvoient découvrir les cordes, et qu'ils ne sçavoient point comment le derriere du theatre estoit disposé. L'un d'eux disoit, c'est une certaine vertu secrete qui enleve Phaëton. L'autre, Phaëton est composé de certains nombres qui le font monter. L'autre, Phaëton a une certaine amitié pour le haut du theatre; il n'est point à son aise quand il n'y est pas. L'autre, Phaëton n'estoit pas fait pour voler, mais il aime mieux voler que de laisser le haut du theatre vuide ; et cent autres resveries, que je m'étonne qui n'ayent perdu de reputation toute l'antiquité. A la fin Descartes, et quelques autres modernes sont venus, qui ont dit : Phaëton monte, parce qu'il est tiré par des cordes, et qu'un poids plus pesant que luy, descend . Ainsi on ne croit plus qu'un corps se remuë, s'il n'est tiré, ou plûtost poussé par un autre corps ; on ne croit plus qu'il monte ou qu'il descende, si ce n'est par l'effet d'un contrepoids, ou d'un ressort ; et qui verroit la nature telle qu'elle est, ne verroit que le derriere du theatre de $\mathrm{l}^{\prime}$ opera $^{21}$.

20 François Hédelin, Abbé d'Aubignac, La Pratique du théâtre, Paris, Sommaville, 1657, livre 4, ch. 9, "des spectacles, machines, et décorations du théâtre», p. 355-385.

21 Bernard le Bovier de Fontenelle, Entretiens sur la pluralité des Mondes, Paris, M. Guérout, 1687, p.15-18. 
Or, c'est exactement ce que montre la planche $X X$ de la $2^{\mathrm{e}}$ série, "Machines», de l'Encyclopédie (Fig. 1), mais à un - important - détail près : $l^{\prime}$ «Élévation en coupe de la charpente en travers des fermes, \& corridor avec un développement de machine de face, nécessaire pour faire venir les décorations du lointain à l'avant scene» n'a de rapport évident ni avec la chute de Phaëton qui nous est montrée dans la vignette de gauche, ni avec la machine à tonnerre que nous voyons sur celle de droite.

En effet, au-delà de la première impression de technicité, prometteuse d'un savoir concret et pratique, les schémas de machines s'avèrent tout à fait insuffisants pour faire comprendre au non-spécialiste comment les dispositifs fonctionnent en réalité, et les textes d'accompagnement n'éclairent guère sa lanterne : «à moins d'avoir fait ou vu fonctionner une telle machine, le lecteur reste nécessairement sur un constat d'échec» reconnaît Jacques Proust ${ }^{22}$, qui conclut que ces planches, où tout semble «transparent, limpide, sans mystère» au point «qu'elles donnent à croire en la parfaite rationnalité du réel», relèvent de l'utopie : «Cette parfaite lisibilité est une illusion, ce charme est celui d'une sécurité trompeuse ${ }^{23}$ ». Diderot n'en promettait d'ailleurs pas plus dans le Prospectus :

Au reste, c'est la main-d'oeuvre qui fait l'artiste ; et ce n'est point dans les livres qu'on peut apprendre à manoeuvrer. L'artiste rencontrera seulement dans notre ouvrage des vues qu'il n'eût peut-être jamais eues, et des observations qu'il n'eût faites qu'après plusieurs années de travail. Nous offrirons au lecteur studieux ce qu'il eût appris d'un artiste en le voyant opérer pour satisfaire sa curiosité ; et à l'artiste, ce qu'il serait à souhaiter qu'il apprit du philosophe pour s'avancer à la perfection.

Elliptiques par dessein, donc, les planches sont également statiques par définition, car elles ne rendent pas compte du mouvement ${ }^{24}$, facteur tout à fait déterminant dans les effets scéniques, ni ne mettent en évidence les principes moteurs. On n'y voit que très rarement les machinistes, qui s'affairaient pourtant nombreux dans les coulisses et les dessous, et jamais les chevaux fournissant l'énergie nécessaire aux déplacements des éléments les plus lourds. Autant dire que ces illustrations démentent la séduisante thèse de Roland Barthes :

22 J. Proust, "Présentation " de L'Encyclopédie Diderot et d'Alembert : planches et commentaires, loc. cit., p. 15 (à propos du métier à bas).

23 Ibid., p. 14-15.

24 Sauf peut-être lorsque - mais de manière très abstraite - le trajet d'un élément mobile est indiqué par une mince ligne en pointillés (planches XI, XII et XIV). 
contrairement aux images modernes, l'homme [est] toujours présent dans quelque coin de la machine $;[\ldots]$ ce qui frappe dans la machine encyclopédique, c'est son absence de secret; ; en elle, il n'y a aucun lieu caché (ressort ou coffret) qui recèlerait magiquement l'énergie, comme il arrive dans nos machines modernes [...]; l'énergie est essentiellement ici transmission, amplification d'un simple mouvement humain [...]. [...] les planches de l'Encyclopédie sont toujours peuplées [...]; vous pouvez imaginer l'objet naturellement le plus solitaire, le plus sauvage ; soyez sûr que l'homme sera tout de même dans un coin de l'image ; il regardera l'objet, le mesurera ou le surveillera, en usera au moins comme d'un spectacle $[\ldots]^{25}$.

Ici, l'absence d'êtres humains est au contraire frappante : les vues en coupe des théâtres nous montrent des salles vides d'acteurs et de spectateurs, qui auraient pourtant été utiles pour nous donner un sens des proportions, par exemple - de manière révélatrice, la seule exception ne représente pas une salle réelle avec de vrais spectateurs, mais le "plan ou projet de la restoration de l'Opéra de Stuttgart», où figure un public virtuel comparable aux figurines dont on peuple les maquettes d'architecture $^{26}$. La présence humaine se limite au plus strict minimum, c'est-àdire aux cas où le dessin d'un mécanisme serait absolument incompréhensible sans les personnes qui le font se mouvoir : ici un «ouvrier faisant le roulement du tonnerre sur un chassis»", là un «Tombeau démoli \& enflammé avec un ouvrier faisant tomber les morceaux de démolition avec un bâton, \& un autre ouvrier jettant sur un flambeau de l'arcanson pour former les éclairs ${ }^{28}$ ». Les occasions ne manquaient pourtant pas de montrer d'autres ouvriers en action, comme par exemple pour actionner le "charriot du tonnerre ${ }^{29}$ " , qui nous apparaît étrangement inerte, ou la manivelle d'un tambour dévidant un tissu qui simule $l^{\prime}$ eau d'une fontaine ${ }^{30}$.

25 Roland Barthes, «Image, raison, déraison», dans L'Univers de l'Encyclopédie, Paris, Les Libraires Associés, coll. «Images d'une civilisation», 1964, p. 11-12.

26 «Théâtres», pl. GG. Remarquons également que, sur ce projet dû à La Guépière, la scène est vide de tout acteur et de tout élément de décor.

$272^{\mathrm{e}}$ section, pl. XI, fig. 3 .

28 Ibid., pl. XIV, fig. 3.

29 Ibid., pl. XX, fig. 3.

30 Ibid., pl. XIX, fig. 3. 
Faut-il voir dans cette volonté d'abstraction un souci de ne laisser planer aucune ambiguïté quant au but instrumental de ces planches, fonction pratique à distinguer de la recherche du beau et de l'émotion dans la représentation du fait théâtral ? L'ambiguïté subsiste pourtant dans ces structures mises à nu, mais dépourvues de sens immédiat, dans ces mécanismes en animation suspendue, montrés mais non expliqués ; les textes d'accompagnement, certes très détaillés, restent purement descriptifs, et non explicatifs, si bien que ces schémas s'assimilent à des écorchés d'anatomie où la forme et la disposition des organes, quoiqu'exactement rendues, ne nous permettent pas d'en déduire le fonctionnement. De telles planches ne s'adressent donc pas vraiment aux facultés rationnelles du spectateur, si bien que leur visée didactique présumée s'en trouve sérieusement mise en doute.

Par ailleurs, l'image veut établir un rapport avec l'expérience esthétique du spectateur à travers les «vignettes», c'est-à-dire les petites images figurant dans les coins supérieurs de dix des planches de la $2^{\mathrm{e}}$ section, et intitulées «idées ou esquisses de décorations». Barthes avait proposé une hypothèse qui peut encore servir de point de départ pour comprendre le rôle de celles-ci :

La plupart de ces planches sont formées de deux parties ; dans la partie inférieure, l'outil ou le geste [...], isolé de tout contexte, est montré dans son essence ; il constitue l'unité informative $[\ldots]$; cette partie de la planche a pour rôle de décliner en quelque sorte l'objet, d'en manifester le paradigme ; au contraire, dans la partie supérieure, ou vignette, ce même objet (et ses variétés) est saisi dans une scène vivante [...] enchaîné à d'autres objets à l'intérieur d'une situation réelle. [...]. En bas, [...] l'instrument n'a aucune vie : inerte, figé dans son essence, il n'est qu'un schème démonstratif $[\ldots]^{31}$.

Si cette explication fonctionne bien pour l'exemple choisi par Barthes (l'atelier du pâtissier), ce n'est pas tout à fait le cas pour les machines de théâtre, à la fois parce que les «objets» figurant dans les vignettes, nous l'avons vu, ne sont pas forcément plus «vivants» que ceux de la planche elle-même, et surtout parce que le rapport entre les deux parties est loin d'être aussi simple, ce qui exclut toute opposition binaire entre «la nomenclature imagée des instruments, ustensiles, produits et gestes», qui «ne comporte par définition aucun secret», et «la vignette, chargée d'un sens disséminé», qui «se présente toujours un peu comme

31 R. Barthes, «Image, raison, déraison», loc. cit., p. 13. 
un rébus $»^{32}$. La différence - essentielle - réside ici dans le caractère illusoire de la «situation réelle» qui donne matière aux vignettes ; car si l'image de la boutique du pâtissier ramène le lecteur à la banalité du quotidien dont la représentation schématique des instruments l'a détourné, les «idées ou esquisses de décorations» l'entrainent au contraire vers l'univers merveilleux de la fable, ou vers la facticité des moyens utilisés pour susciter la magie au théâtre : ce n'est pas vraiment Phaëton en train de tomber, ni Médée en train de s'envoler que nous montrent les vignettes, contrairement à celles du pâtissier, où ce sont de véritables ouvriers au travail que nous voyons.

N'en concluons pas pour autant que ces images projettent le lecteur hors de l'expérience commune, car elles illustrent un nombre très limité d'effets à machine, appliqués à des œuvres-phare du répertoire lyrique (Armide, Médée, Phaëton...). Scènes familières donc pour quiconque fréquentait l'opéra de Paris dans la première moitié du siècle ; scènes génériques surtout dans la mesure où ces éléments décoratifs n'appartiennent pas à un titre en propre. Ainsi, la vignette montrant l'épisode célèbre de la chute du char de Phaëton peut évoquer, outre la tragédie en musique de Quinault et Lully (1683), une comédie hérö̈que de Boursault pour le Théâtre-Français (1691), une comédie de Palaprat pour l'ancien Théâtre-Italien, Arlequin Phaëton (1692) ${ }^{33}$, ainsi que plusieurs parodies en vaudevilles au nouveau Théâtre-Italien ${ }^{34}$. Les similitudes que l'on peut observer entre les frontispices de Phaëton pour les éditions de 1683 et de 1692 et l'«esquisse de décoration» de l'Encyclopédie suggèrent qu'au milieu du XVIII ${ }^{\mathrm{e}}$ siècle, le clou du spectacle, tout comme l'envol du char de Médée, était devenu un poncif visuel - et le choix qu'en fait Fontenelle pour illustrer les progrès de la philosophie mécaniste atteste de sa diffusion auprès d'un large public, $\mathrm{d}^{\prime}$ 'où sa valeur de synecdoque pour l'ensemble des effets scéniques. On aurait grand'peine à établir un

32 Id. : "A son étage démonstratif, la planche encyclopédique constitue une langue radicale, faite de purs concepts, sans mots-outils ni syntaxe ; à l'étage supérieur, cette langue radicale devient langue humaine, elle perd volontairement en intelligible ce qu'elle gagne en vécu".

33 Publiée dans Le Théâtre Italien de Gherardi, ou Recueil général de toutes les Comédies et Scènes Françoises jouées par les Comédiens Italiens du Roy, pendant tout le temps qu'ils ont été au Service, Paris, J.-B. Cusson et P. Witte, 1700, vol. 3, avec un frontispice qui démarque celui des éditions de l'opéra de Quinault et Lully.

34 Celles de l'abbé Macharty (1721), de Romagnesi et Dominique (1731) et de Riccoboni (1743). Aucune n'a été publiée. 
décompte des ouvrages où pourraient figurer les autres éléments de décor, moins spécifiques encore, répertoriés dans l'Encyclopédie.

Résumons : d'un côté, des schémas inexploitables en pratique, car insuffisants pour faire comprendre dans son intégralité le fonctionnement des machines (à moins de posséder déjà le savoir technique adéquat); de l'autre, des vignettes qui non seulement illustrent des décors et des trucages banals, mais possèdent une faible valeur esthétique et, surtout, ne livrent pas, à une exception près ${ }^{35}$, la clé du processus permettant de réaliser l'effet tel que le spectateur le voit sur scène. Entre l'écorché mécanique et le tableau, point de causalité évidente, ni de simple lecture, comme le postulait Barthes ${ }^{36}$ : ce sont en fait deux signifiants hétérogènes appartenant à deux codes distincts.

Le schéma ne remplit pas la promesse implicite de révéler l'arcane des mécanismes ; c'est un document factice, un signifiant dont le référent est l'image mentale que le lecteur/spectateur peut se faire d'un «schéma technique» : dument impressionné par la précision des détails, celui-ci s'imaginera que ces planches pourraient éventuellement servir à construire de véritables machines fonctionnelles, et il perdurera dans son aveuglement tant qu'il n'essaira pas de passer à l'acte. La vignette, en revanche, relève d'un code esthétique qui suscite une image ostensiblement fausse : représentation non de ce qu'on a effectivement montré et vu sur scène, mais de l'image mentale que le spectateur doit s'en faire, voire même représentation iconographique de l'action fictive en soi. A partir d'une même réalité - l'instance performative - se créent ainsi deux images mentales, également infidèles : l'une efface toute trace de la machine qui produit l'effet, tandis que l'autre, prétendant au contraire nous dévoiler cette machine, ne nous en fournit qu'un ersatz de description.

Que l'on examine par exemple la planche XII, montrant l'«élévation en coupe de la charpente et des corridors avec le développement d'une machine de travers et idées ou esquisses d'un antre infernal, d'un volcan enflammé» (Fig. 2), et le texte censé l'expliquer :

35 La figure 3 de la planche XIX, déjà mentionnée, qui nous laisse voir le tambour à manivelle simulant l'eau d'une fontaine.

36 Lecture analytique (haut $>$ bas) ou synthétique (bas $>$ haut). R. Barthes, «Image, raison, déraison", loc. cit., p. 14. 
Fig. 1. Elévation en coupe de la charpente du comble \& des corridors, avec le développement d'une machine de travers. A, A, élévation du mur portant les fermes du comble. B, développement d'une machine faisant le vol de la machine montant d'un côté \& descendant de l'autre. a, o, b, est la révolution de la machine. C, cordage traversant le travers du théâtre d'un corridor à l'autre, servant de conduit aux chars. D, char dans lequel passent les fils qui portent la machine. E, E, fils portant la machine, qui sont enveloppés sous le tambour F. G, tambour de renflement sur lequel est enveloppé un cordage $\mathrm{H}$, servant de retraite à la main pour lâcher la machine. I, retraite pour lâcher le char. $K$, développement du char D. L, cordage servant de conduit au char. $M$, retraite du char servant aux contrepoids. N, N, corridors pour le service des machines. $\mathrm{O}, \mathrm{O}$, corridors où sont posés les treuils pour les contrepoids. $\mathrm{P}, \mathrm{P}$, treuils servant à monter les contrepoids. $\mathrm{Q}$, plancher du ceintre où sont les machines. $R$, second plancher des tambours des machines. Fig. 2. Décoration de l'intérieur d'un antre avec l'apparition de Jupiter commandant aux esprits infernaux. 3. Extérieur d'un volcan avec flammes \& rochers précipités.

On pourra peut-être, avec quelque effort, saisir le fonctionnement général de ce dispositif permettant ce qu'on appelle un «vol transversal oblique» ; mais l'absence de nacelle, normalement suspendue au «char» (la pièce $\mathrm{D}$ qui figure également en gros plan), rend malaisée la visualisation de la machine in vivo et ce d'autant plus que les vignettes 2 et 3 semblent n'avoir aucun rapport avec elle, ainsi que je l'ai fait remarquer plus haut à propos de la planche XX. Et justement, c'est plutôt à la chute de Phaëton que pourrait correspondre cette machine à vol oblique...

Comment donc définir le statut de ces images, et surtout le rapport entre $l^{\prime}$ illustratif et $l^{\prime}$ explicatif ?

Catherine Kintzler a démontré que la tragédie lyrique obéissait à une poétique sui generis parfaitement cohérente et adoptant la doctrine néoaristotélicienne ${ }^{37}$, et nous pouvons considérer les éléments représentés sur les vignettes comme les unités minimales d'une poétique visuelle du spectacle dramatique, complémentaire de celle qui régit le texte. Le système se trouve ici réduit à sa plus simple expression, car le florilège

37 Catherine Kintzler, Poétique de l'opéra français de Corneille à Rousseau, Paris, Minerve, 1991. 
qu'offre l'Encyclopédie ne prétend pas à l'exhaustivité : les machines illustrées sont celles qui s'intègrent au bâtiment de manière permanente, à l'exclusion des éléments autonomes et des pratiquables (comme par exemple ces monstres habités dont Berain nous a laissé des croquis explicatifs très éclairants), mais aussi des costumes et des panneaux décoratifs peints.

Ce système concerne les changements de décor par mouvement des châssis et des fermes, les vols (simples ou obliques), les descentes et montées «en gloire», et les effets de feu et d' $\mathrm{eau}^{38}$; ces trucages déterminaient en grande partie le choix des sujets (mythologiques de préférence), le changement quasi obligé de décor à chaque acte (si possible opéré «à vue») et l'inclusion de "scènes à faire» mettant en œuvre les machines disponibles : apparitions et disparitions de créatures surnaturelles, catastrophes (tempêtes comme dans Alcyone de Marais et Houdar de la Motte, embrasement d'un palais comme dans Armide de Quinault et Lully, éruptions volcaniques comme dans les Indes galantes de Fuzelier et Rameau...), sortilèges divers et scènes marines. Les planches dressent ainsi le catalogue des lieux communs scénographiques à partir desquels les auteurs devaient agencer leur matière dramatique ; c'est un idiome vieux de presque deux siècles qui trouve ici son ultime transcription, alors qu'il est en plein déclin.

La démarche des éditeurs encyclopédistes semble en effet moins ambiguë si l'on considère que l'illustration des machines de théâtre se place à un tournant historique, lorsque la machinerie conventionnelle du système à l'Italienne s'est depuis longtemps pétrifiée dans un répertoire de procédés qui ne varient plus pour l'essentiel, alors que l'opéra s'est déjà orienté vers un certain «réalisme» (prôné par Diderot) et que l'on constate la disparition progressive de la tragédie lyrique fondée sur le merveilleux issu de la mythologie, de la magie, ou même de phénomènes naturels extraordinaires. Pourtant, d'autres effets étaient inventés, d'autres manifestations du surnaturel fascinaient le public, trouvant leur champ d'expression dans les spectacles forains, les fantasmagories, le ballet, la pantomime, et plus tard le music-hall, le cirque ou le GrandGuignol - mais seules les machines de l'opéra, en dépit de leur caractère suranné, furent jugées dignes de ce dictionnaire qui se voulait «général». Ce traitement du théâtre, qui écarte les genres «mineurs», montre les tensions conceptuelles entre la démarche encyclopédique vouée à la

38 Voir notre typologie dans Le Jeu de l'Ordre et du Chaos: Comédie et pouvoirs à la Fin de règne, 1673-1715, Paris, Honoré Champion, coll. "Lumière classique ", 2002, p. 361-380. 
valorisation des arts mécaniques englobant l'humble machine-outil, et le rouage magique nécessaire aux spectacles de la meilleure des sociétés.

En 1772, il ne restait plus sur la scène de l'opéra de prodiges à démystifier, et les schémas désormais inutiles de l'Encyclopédie, s'ils ont le "charme [...] de donner à rêver ${ }^{39}$ ", sont loin de constituer un document vivant de la pratique théâtrale de leur époque : ils offrent au lecteur l'image nostalgique et figée d'un imaginaire classique qui, au moment où l'on publiait le dixième volume de planches, était déjà irrémédiablement voué à la disparition.

\section{GUY SPIELMANN}

Georgetown University (Washington)

39 J. Proust, «Présentation», loc. cit., p. 15. 
$2^{2}$ Jection

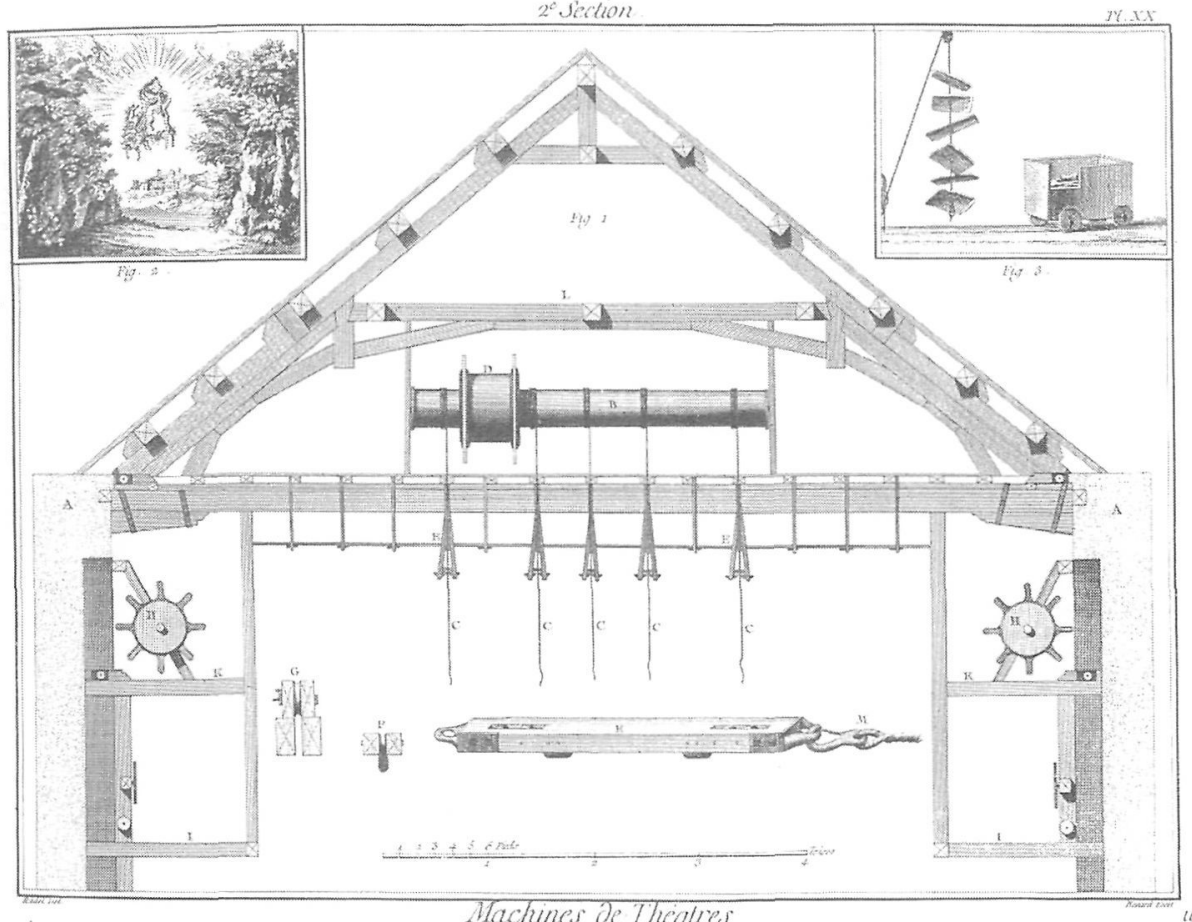

Machunes de The alles

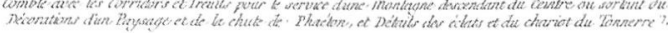

Figure 1. 


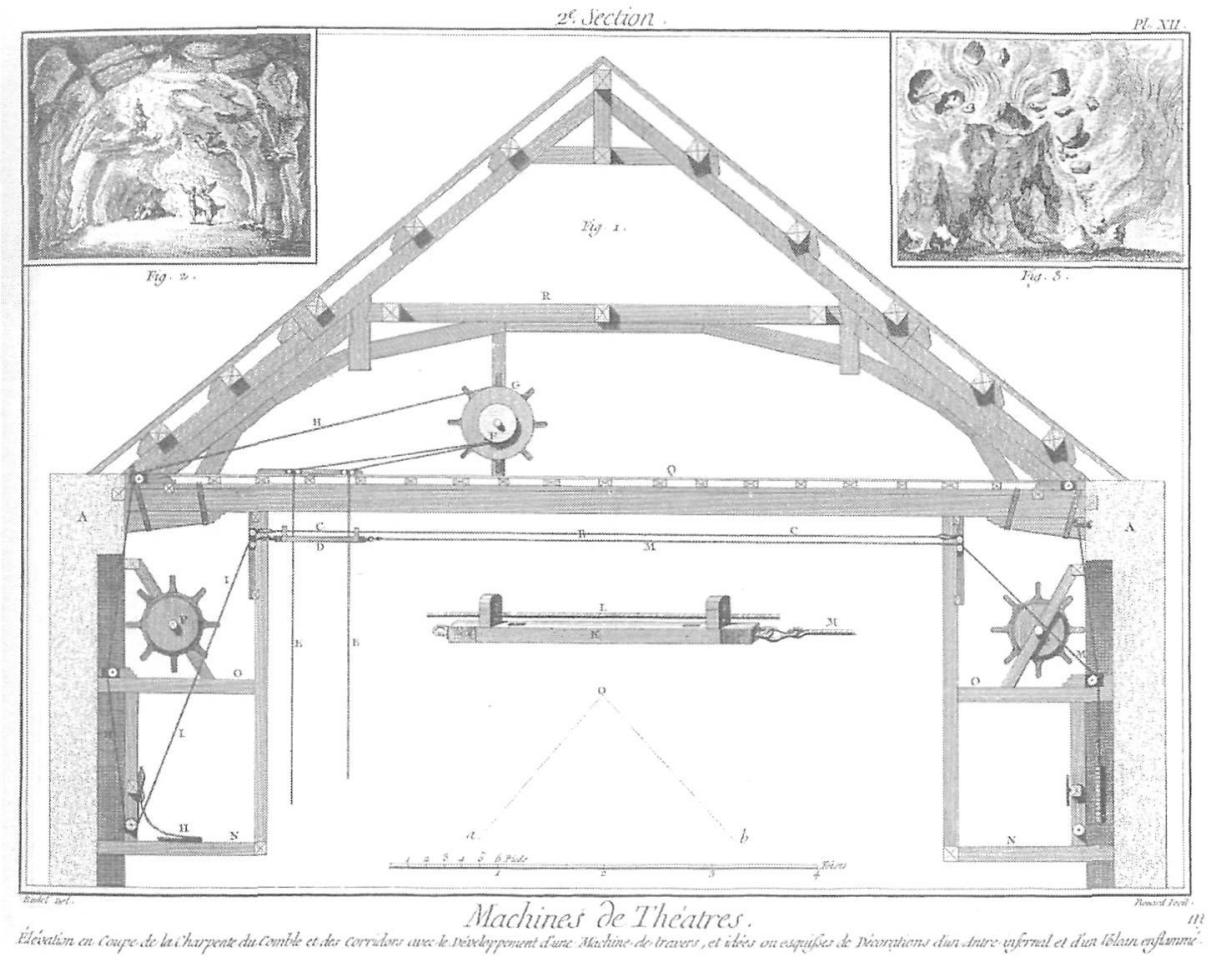

Figure 2. 